\title{
The novel epiligament theory: differences in healing failure between the medial collateral and anterior cruciate ligaments
}

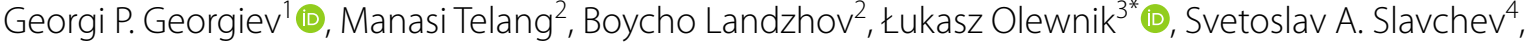 \\ Robert F. LaPrade ${ }^{5}$, Kacper Ruzik ${ }^{6}$ and R. Shane Tubbs $7,8,9,10,11$
}

\begin{abstract}
According to current literature, $90 \%$ of knee ligament injuries involve the medial collateral ligament or the anterior cruciate ligament. In contrast to the medial collateral ligament, which regenerates relatively well, the anterior cruciate ligament demonstrates compromised healing. In the past, there were numerous studies in animal models that examined the healing process of these ligaments, and different explanations were established. Although the healing of these ligaments has been largely investigated and different theories exist, unanswered questions persist.

Therefore, the aim of this article is 1 ) to review the different historical aspects of healing of the medial collateral ligament and present the theories for healing failure of the anterior cruciate ligament; 2) to examine the novel epiligament theory explaining the medial collateral ligament healing process and failure of anterior cruciate ligament healing; and 3) to discuss why the enveloping tissue microstructure of the aforementioned ligaments needs to be examined in future studies.

We believe that knowledge of the novel epiligament theory will lead to a better understanding of the normal healing process for implementing optimal treatments, as well as a more holistic explanation for anterior cruciate ligament healing failure.
\end{abstract}

Keywords: Epiligament, Medial collateral ligament, Anterior cruciate ligament, Healing, Novel theory

\section{Introduction}

One of the most commonly injured ligaments in the knee is the medial collateral ligament (MCL) [1-4]. Most MCL injuries are the consequence of external rotation, valgus loading, or a combined force vector in sporting activities such as football, skiing, and ice hockey. They are mostly isolated and occur predominantly in young athletes $[3,5]$. They are typically associated with mediolateral instability, especially during cutting or pivoting maneuvers [5]. It is now well established that most knee ligament injuries involve the MCL or the anterior cruciate ligament (ACL).

\footnotetext{
*Correspondence: lukasz.olewnik@umed.lodz.pl

${ }^{3}$ Department of Anatomical Dissection and Donation, Chair of Anatomy and Histology, Medical University of Lodz, Łódź, Poland

Full list of author information is available at the end of the article
}

The incidence of MCL injury has increased over recent decades and is frequently encountered in modern sports medicine [5]. After injury, the ligaments do not heal by regeneration but by formation of scar tissue, similar to other wound healing models [6-8]. Many studies have shown that while the MCL can heal fairly well, it cannot be fully restored. Therefore, different treatment options, such as tissue engineering approaches, nonsteroidal antiinflammatory drugs, local corticosteroids, hyperbaric oxygenation, growth factors, ultrasound or electrical stimulation, laser therapy, and gene therapy have been attempted [9-12]. Unlike the MCL, the ability of the ACL to heal spontaneously is inadequate. In the past, numerous experiments in different animal models have been performed, and different theories have been proposed to 
explain the healing differences in these ligaments; however, questions regarding the superior healing process of the medial collateral ligament and its failure in injuries of the anterior cruciate ligament still exist $[1,5,13-16]$. It should be noted that all the morphological explanations about the healing process of these ligaments in animal models investigate only the extracellular matrix in both normal and injured ligaments, and none of them considers the enveloping tissue of the ligament, termed epiligament (EL) [3, 11, 12, 17].

After extensive research on epiligament morphology of the MCL in rats and humans in both normal conditions and injury, as well as investigation of the same tissue in the ACL and comparison between these two commonly injured ligaments, a novel EL theory was proposed by Georgiev et al. [6-8, 11, 12, 18-22].

The aim of the current paper is to review the current literature concerning the knowledge of commonly injured ligaments of the knee and the differences between MCL and ACL healing. We discuss in detail how this new theory clearly explains the worsened capacity for healing of the ACL. We also emphasize why future investigations for better advances in ligament repair treatment should be directed to the epiligament.

\section{MCL anatomy}

The MCL is an intricate structure that acts as the primary static stabilizer of the knee joint, counteracting rotation caused by a valgus force $[1,23]$. It comprises three constituents: the superficial MCL (sMCL), the deep MCL $(\mathrm{dMCL})$ and the posterior oblique ligament (POL) [5, 24]. The largest ligament in the medial aspect of the knee is the sMCL, which connects the medial femur and tibia through one femoral and two tibial attachments [5]. The thickened medial portion of the joint capsule is represented by the dMCL and has two parts, the meniscofemoral and meniscotibial ligaments [5]. The posteromedial aspect of the joint capsule is attached to and reinforced by fibrous extensions from the main common tendon of the semimembranosus, which constitute the POL $[5,24]$.

\section{$\mathrm{ACL}$ anatomy}

The ACL arises from the distal femur and attaches to the anterior intercondylar area of the tibia [25]. It is composed of two bundles, anteromedial and posterolateral, with different tibial attachments [26-28]. Excessive anterior translation and internal rotation of the tibia relative to the femur is resisted by the ACL $[14,25]$. Studies indicate that the anteromedial bundle is primarily responsible for resistance to anterior tibial translation, while the posteromedial bundle contributes to the control of tibial rotational laxity [26].

\section{Ligament microstructure}

Ligaments are hypocellular and hypovascular structures built of dense regular connective tissue [29-31]. Collagen is the most widespread extracellular component of soft connective tissue and is the major tensile-bearing element $[6,7,31,32]$. The endoligament is sheath of connective tissue that covers the collagen fibers in the ligament proper, which are organized into fascicles $[6-8,11,12,22$, 33]. Collagen constitutes approximately $75 \%$ of the dry weight of ligaments, the predominant type being type I, which accounts for nearly $85 \%$ of the total collagen of ligaments and is chiefly responsible for their tensile strength $[6,7,32,34]$. The remaining $15 \%$ includes types III, V, VI, $\mathrm{XI}$, and XIV [34]. Type III is involved in ligament repair, and its synthesis is significantly increased after grade III ligament injuries [9, 30, 34]. The role of collagen type III in proper ligament recovery has been discussed previously [30,35]. Its synthesis swiftly increases during the first stages of ligament healing and during ligamentization after tendon grafting, exceeding the level of synthesis of type I. Rates typically return to normal by 52 weeks after injury [30, 35]. Hauser et al. [34] found that after injury, fibroblasts mainly synthesize collagen type III. Considering the large number of fibroblasts in the EL, it is reasonable to suggest that they are chiefly responsible for the upregulation of collagen type III.

According to Yang et al. [36], collagen type III is also essential for generating tissue matrix, fetal tissue matrix, and scars. These authors proposed that the ability of collagen type III to crosslink by disulfide bridges contributes to its favorable deposition in sites of tissue regeneration. Amiel et al. [37] also established that collagen type III increased during ligamentization after tendon grafting.

On the other hand, collagen type $\mathrm{V}$ engages in the organization of collagen type I fibrils and the regulation of their diameters, which also occurs during ligament healing [6, 7, 35, 38]. According to Breuls et al. [38], type $\mathrm{V}$ collagen fibrils participate in the regulation of extracellular matrix modeling and remodeling by controlling collagen fibril initiation. Collagen type XIV is involved in linear fibril growth [30].

Collagen fibers in ligaments are organized into fascicles enveloped by a thin connective tissue sheath known as the endoligament. The endoligament in turn is connected to a more vascular connective tissue layer that covers the entire ligament, termed the epiligament (EL) [8, 20,33].

\section{Structure of the epiligament}

Surface layers of connective tissue are characteristically associated with bone, cartilage, striated muscle, nerves, and tendons. These layers are termed periosteum, perichondrium, epimysium, epineurium, and epitendon, 
respectively [39]. Bray et al. [40], in 'Fine vascular anatomy of adult rabbit knee ligaments', provided the first definition of 'epiligament' (epi- [Greek - on or upon]; ligament [Latin - ligare, to bind]). This structure has been described as 'surrounding adherent connective tissue removed simultaneously with the ligament but ... grossly distinguishable from ligament tissue proper' [40]. Later, Chowdhury et al. [33] also examined the external surface of the EL of the MCL in rabbits and described two types of cells, fibroblasts and spinous adipocytes. Apart from covering the ligament tissue, the EL merges with the periosteum at the sites of ligament insertion [29]. In contrast to the ligament, the EL contains multiple cell types such as fibroblasts, fibrocytes, adipocytes, and blood vessels $[6-8,11,12,18-20,33,41]$. Georgiev et al. [21] presented the ultrastructural characteristics of these cells. Fibroblasts have been described as large and well formed; they display a very delicate chromatin structure with a prominent nucleolus. The cytoplasm contains free ribosomes, polysomes, a well-presented rough endoplasmic reticulum, a poorly developed Golgi apparatus, spherical mitochondria, and single lysosomes. The second cell type, spindle-shaped fibrocytes, were described as having large vacuoles and eccentric, flat nuclei surrounded by a basal lamina and rough endoplasmic reticulum. In the intercellular space, Georgiev et al. also found collagen fibers with multiple orientations, as well as myelinated and unmyelinated nerve fibers and blood vessels. Similar ultrastructural characteristics of fibroblasts and fibrocytes, as reported above, were confirmed by Georgiev et al. [8] in humans. They finally concluded due to the ultrastructural characteristics that fibroblasts might be involved in differentiation, phagocytosis, and collagen synthesis; the authors also hypothesized that single collagen fibers or those grouped in bundles may respond to ligament tension in different directions $[8,21]$. With the aim of obtaining more detailed knowledge of the MCL and ACL ELs and comparing it in rats, Iliev et al. [32] found a statistically significant difference in the number of cells per $\mathrm{mm}^{2}$ in the EL of the two ligaments, with a greater number in the MCL. Furthermore, the EL contains an abundance of blood vessels and sensory/proprioceptive nerve elements, which form a complex network $[6-8,11,12$, 18-20, 33]. It has been proposed that the EL has a role in ligament growth and healing and may control water and metabolite influx into the ligament $[33,40]$. Several studies have pointed toward the EL as a donor of fibroblasts and other connective tissue cells, progenitor cells, and blood vessels, which migrate toward the body of the ligament via the endoligament and are crucial for ligament repair $[6-8,11,12,18-21,41]$. These studies clearly indicate that during early healing, EL tissue formed new granulation tissue after injury via the endoligament and thus is the main donor of cells and blood vessels for the repair process. Georgiev et al. [12] statistically analyzed the number of cells in the EL-ligament scar, which demonstrated no difference between spontaneous healing and healing after suture application. The reported histological data on the EL's main role in ligament repair and the proposal of a new EL theory could be used as a basis for the development of new treatment regimens with improved patient outcomes.

The collagen fibrils in the EL mainly comprise collagen types III and V $[6,7,9,22,32]$. Collagen type III is integral to ligament repair $[9,30,34]$. Collagen type $\mathrm{V}$ is also associated with ligament recovery, and the intensity of its expression corresponds to the diameter of collagen fibrils [35]. In the MCL and ACL, the immunohistochemical expression of the aforementioned collagen types was stronger in the EL than in the ligament proper and was greater in the EL of the MCL than in the ACL [32]. Fibroblasts are also responsible for the synthesis of matrix metalloproteinases (MMPs), decorin, fibronectin, and fibromodulin, which are involved not only in the degradation of the ligament after injury but also in subsequent cell proliferation and ligament remodeling $[6-9,11,12$, 18-22]. Georgiev et al. [20] reported that the enzymatic activity of MMP-2 and MMP-9 was greater in the EL of the MCL than in the same structure of the ACL and speculated the important role of these enzymes in the normal function and difference in the healing potential of these ligaments.

\section{Ligament healing morphology}

In a dog model, O'Donoghue et al. [42] reported that surgical treatment led to a decrease in the amount of newly synthesized tissue and accelerated collagenisation in the healing process, thus improving morphological characteristics. Hart and Dahners [43] and Hildebrand and Frank [44] found no statistically significant difference in improved endurance of the MCL between nonoperative treatment and treatment by suturing. According to Chimich et al. [45], bringing the ends of the ruptured ligament closer together has a definite advantage during the granulation and remodeling stages since it improves the macroscopic, histological, and biomechanical properties of the ligament. According to the authors, the shorter distance between the two ends approximated by suturing is associated with faster filling of the space between them with newly synthesized tissue and with more rapid remodeling. Nevertheless, in an experimental rabbit model, Chimich et al. [45] demonstrated that there was no statistically significant difference in the strength of the ligament after 40 weeks between animals treated surgically and those treated conservatively. These authors also noted a number of similarities between the models of the 
two types of treatment: healing is mediated by granulation tissue, which is macroscopically and histologically different from normal tissue; approximately 3 weeks after injury, the neoligament is characterized by hypercellularity and is mixed with other healthy tissues; in comparison with the contralateral ligament at 14 weeks, the laxity of the injured ligament appears restored; the newly formed connective tissue is shortened, which is also observed in other connective tissue models; remodeling of the tissue continues over time, but the macro- and microscopic appearances of a regenerating tissue are evident even after 40 weeks; in both models, approximately $65 \%$ of the strength of the ligament is restored after the 40th week compared to the contralateral ligament.

Loitz-Ramage et al. [46] compared the outcomes of conservative and operative treatments in a rabbit model over a longer period of time. The authors compared the results of treating an $8 \mathrm{~mm}$ gap between the ends of the torn ligament with or without a Z-suture and found that reducing the distance to $4 \mathrm{~mm}$ using the suture generated greater strength than was achieved at $8 \mathrm{~mm}$. Extrapolating this result to clinical practice, the authors stated that patients with longer distances between the two ends of the torn ligament, for instance in those patients in which adjacent bones are dislocated or ligament ends are retracted, could restore normal function through low or moderate loading, but there is a significant risk of re-rupture in the event of heavy loading. Using their rabbit model, McDougall et al. [47] reported that levels of angiogenesis during the sixth week after surgical treatment corresponded to those in control animals that had undergone placebo surgery. The authors suggested that operative treatment with suture application could have accelerated the healing process so much that angiogenesis had already been reversed; otherwise, the repositioning of the ends of the injured ligament might have hindered neovascularization.

Ishiguro et al. [48] reported the presence of promatrix metalloproteinase-9-positive cells in the perivascular area of the ruptured ACL and promatrix metalloproteinase-2-positive cells between irregular collagen bundles in the stumps of this ligament. The authors could not determine whether the positive reaction of these MMPs was due to rapid degradation or the result of prior degradative changes. Creighton et al. [10] suggested that surgical treatment would decrease the maximum distance between the two ends of the torn ligament with the intent of improving healing if rupture of the ligament was incomplete. The tear would thus be shortened, and the ligament would be realigned in a state close to its anatomical one [47].

After comparing the expression of different MMPs in the MCL and the ACL, Zhou et al. [49] concluded that numerous MMPs could be associated with differences in healing potential. They reported that fluorescent MMP-2 activity was higher in the injured ACL than in the MCL, which could be one reason for ACL healing failure. Majima et al. [17] compared cyclic creep between surgically repaired and nonrepaired ligaments and found no significant difference; regardless of whether the ligament was operated on, creep was 3-4 times that of a normal ligament 6 weeks after injury. The authors concluded that acute ligament repair did not alter the potential of the healing complex to creep relative to controls, even at 6 weeks after injury. According to Tang et al. [50], MMP-2 expression in fibroblasts of the injured ACL was 6.3 times higher than that of the injured MCL. MMP-9, in contrast, was upregulated in the injured MCL but to a much lesser degree than in the injured ACL. Zhang et al. [51] reported higher mRNA levels of MMP-1, MMP-2, MMP-14, MMP-17, MMP-23A, MMP-23B and TIMP-4 in MCL fibroblasts than in ACL fibroblasts. Furthermore, it was suggested that the differential expression of MMPs between the MCL and ACL could partly account for the differential healing potentials of the two ligaments. Georgiev et al. [19] observed the distribution and expression of MMP-2 in normal rat tissue and during healing after acute injury. They showed that fibroblasts in the EL of the MCL normally generated low levels of MMP-2. After grade III injury, high levels of MMP-2 were expressed during early ligament healing. Georgiev et al. [12] were the first to describe the ultrastructural changes in the EL of the MCL during the first month of ligament healing after injury. On the eighth day after injury, intensive angiogenesis was observed in the EL; the granulation tissue between the transected regions was hypercellular and represented mainly by EL fibroblasts and progenitor cells migrating through the endoligament. On the sixteenth day, diminished angiogenesis in the EL was observed, with less distinguishable granulation tissue and hypercellular tissue but a better organization pattern of EL-ligament scarring; the cells of the EL also migrated through the endoligament. On the thirtieth day after injury, the healing process advanced, and the EL tissue was similar to controls. In conjunction with the light microscopic study, the authors also presented in detail the ultrastructural characteristics of the fibroblasts in the EL, with well-formed large nuclei and clearly visible heterochromatin; the cytoplasm had well-developed rough endoplasmic reticulum, poorly developed Golgi apparatus, free ribosomes, polysomes, mitochondria, phagocytic vacuoles and lysosomes. All these characteristics define these cells as metabolically active structures during the healing process. Finally, light and electron microscopic observations showed no difference in the structure of the ligament during spontaneous healing and after suture 
application, as indicated by the numbers of cells in the EL ligament scar tissue. According to the authors, the limitations of this animal model were as follows: (1) all injuries were induced by scalpel transection, not an ideal simulation of common traumatic injuries; (2) only the EL of the mid-substance of the MCLTCL was studied; (3) the distance between the transected edges was no more than $1.5 \mathrm{~mm}$; and (4) the statistical analysis used nonparametric tests, which generally have lower power. To attempt to mitigate this, the authors used two types of tests, the Kruskal-Wallis $\mathrm{H}$ test and Mood's median test.

\section{Different explanations of ACL healing failure}

Much is known about the different healing potentials of the ACL and MCL $[1,5,14-16]$. The ACL has poor healing ability, in contrast to MCL $[1,5,14]$. However, the reasons for the difference are not entirely clear, but multiple explanations have been suggested.

One explanation points to the fact that the ACL has an intra-articular location and is exposed to synovial fluid, which inhibits fibroblast function [52]. Another attributes it to the different ultrastructures of the connective tissue cells in the MCL and ACL [53]. More recent studies showed differences in fibroblasts and their proliferative potential between the ACL and MCL [54]. It has been suggested that higher levels of nitric oxide produced by ACL cells cause collagen inhibition and proteoglycan synthesis [55]. Others have noted a stronger blood flow and more pronounced angiogenesis in the MCL after injury, followed by accelerated healing [56]. MMP-2, -9 , and -13 expression is also reported to differ $[20,57]$. The failure of cells and blood vessels to adequately bridge the gap between the ruptured ends of the ACL could be one reason for the unsatisfactory healing of this ligament [58]. Vavken and Murray [59] suggested that plasmin circulating in synovial fluid slows healing by breaking down the fibrin clot. Moreover, the intra-articular environment reduces biological and mechanical support by adjacent tissues, meaning that cells and blood vessels are less likely to bridge the gap between the ligament's ruptured ends properly [59].

The existence of these theories explaining ACL failure healing has shown that this process is "multifactorial" and that no one theory can explain the reasons for an inadequate healing process. A novel theory amplifying the others, describing one single unit ligament complex (target for future treatment strategies) and that could be responsible for healing or healing failure, would be ideal.

\section{Novel epiligament theory}

Several reports by Georgiev et al. [6-8, 11, 12, 18-22] have presented the EL as a donor of fibroblasts, progenitor cells, and blood vessels, which migrate via the endoligament toward the ligament body. The authors accept its key role in ligament function and healing. Their recent studies [12, 18, 19] of MCL injury models have revealed that the fibroblasts in the EL are not static but are responsible for synthesizing various types of collagen, matrix metalloproteinases, decorin, fibronectin, and fibromodulin. All these molecules are implicated in the degradation, proliferation, and remodeling of the ligament after trauma [12, 18, 19, 32].

After investigations of the EL in rats, this tissue has been studied in humans. To confirm its supposed role and to account for ACL healing failure, Georgiev et al. [22] presented the morphologies of the ELs of human MCL and ACL. The authors established that the EL of the MCL and ACL in humans was quite different from the morphology of the ligament substance, which confirmed data of EL morphology in the rat. The EL of the MCL and ACL was comprised of fibroblasts and fibrocytes, adipocytes, collagen fibers and neurovascular bundles. This morphological description confirms the similarity of the EL between humans and rats. Additionally, the number of cells enumerated during light microscopy revealed that fibroblasts in the EL of the MCL were greater than those in the EL of the ACL. After comparative quantitative analysis of the number of cells, the authors established that the mean number of cells in the EL of the MCL was $32 \%$ greater than that in the EL of the ACL; the EL passed through the endoligament to the ligament proper, and in comparing these structures, the authors established that the endoligament of the MCL contained 36\% more cells than that of the ACL. A greater incidence of cells per $\mathrm{mm}^{2}$ in the EL was reported in the MCL than in the ACL. These data confirmed previous literature data describing the similar predominance of cells in the EL of the MCL as compared to the same structure of the ACL in the rat. After the presented quantitative analysis, Georgiev et al. [22] suggested that further reasons for the discrepancy in healing potential could be the differences in the expression of collagen types I, III, and V in the ELs and ligament proper.

After evaluation of the immunohistochemical expression of the aforementioned collages, the authors observed that in the EL, immunostaining for collagen type I is localized predominantly in the tunica media of the blood vessels and in the ligament proper of both ligaments, where immunoreactivity was expressed ubiquitously and appeared moderate. For procollagen type III, a positive immunoreactivity was observed in the adventitia of blood vessels and the periphery of adipocytes and was stronger in the EL of the MCL. Expression was also strong in the ligament proper of the MCL in contrast to the ACL, where it was low or absent. The authors report that collagen type $\mathrm{V}$ was detected in the tunica media 
of blood vessels and in the superficial layer of the EL in both ligaments. For precise evaluation of expression, a semiquantitative IHC profiler was performed. Finally, the authors reported that (1) there are fewer connective tissue cells in the EL of the ACL than in the MCL, and the difference is statistically significant; (2) the expression of collagen types I and V and procollagen type III is higher in the EL of the MCL than in the ACL; and (3) procollagen type III is also expressed in the ligament tissue of the MCL but not in that of the ACL under physiological conditions.

According to Georgiev et al. [22], fewer cells in the EL of the ACL than in the MCL in the healthy knee cannot ensure an adequate healing capacity. Moreover, the expression of collagen type I (accounting for ligament tensile strength), procollagen type III (integral to proper ligament healing), and collagen type V (organizing collagen type I fibrils and regulating their diameters) in fibroblasts is also lower in the EL of the ACL than in the MCL. All aforementioned variations in EL morphology and differences in fibroblast activity in the healthy knee, especially the expression of procollagen type III, provide additional explanations for the failure of ACL healing after trauma.

Finally, the authors concluded that the ELs of the $\mathrm{MCL}$ and the ACL are quite different from the ligaments proper. They contain abundant fibroblasts, fibrocytes, and adipocytes as well as neurovascular bundles. EL fibroblasts are not static cells and produce various quantities of collagen types I, III, and V. Immunologically, expression of the collagen types studied was higher in the EL than in the ligament proper and higher in the EL of the MCL than in the ACL. In addition, there was a statistically significant difference between the numbers of cells per square millimeter in the ELs of the two ligaments that was higher in the MCL. Thus, Georgiev et al. have presented new data on the structural and functional significance of EL tissue that suggests it could be responsible for the better healing capacity of the MCL over the ACL.

\section{Conclusion}

In the current literature review, we present what is known about ligament healing in the MCL. We also present commentary on this topic to draw attention to the novel EL theory concerning a novel presentation of the healing process regarding the newly formed tissue filling the gap between the ends of the ruptured ligament. We emphasize that future investigations for better ligament restoration should be directed at the EL because it contains the major substrate of cells and blood vessels responsible for ligament healing. The novel theory, based on the number of cells and vessels and the distribution of collagens and matrix metalloproteinases, could simply and clearly explain the worsened capacity of ACL healing. This new theory further developed existing theories based on differences in fibroblasts, impaired blood flow and MMP expression. This could be a starting point for new treatment strategies. The future will reveal whether this novel hypothesis will be accepted by morphologists and knee surgeons and whether it will be embraced as another explanation of ACL healing failure.

\section{Authors' contributions}

The author(s) read and approved the final manuscript.

\section{Competing interests}

No conflict of interest.

\section{Author details}

${ }^{1}$ Department of Orthopedics and Traumatology, University Hospital Queen Giovanna-ISUL, Medical University of Sofia, Sofia, Bulgaria. ${ }^{2}$ Department of Anatomy, Histology and Embryology, Medical University of Sofia, Sofia, Bulgaria. ${ }^{3}$ Department of Anatomical Dissection and Donation, Chair of Anatomy and Histology, Medical University of Lodz, Łódź, Poland. ${ }^{4}$ University Hospital of Orthopedics "Prof. B. Boychev", Medical University of Sofia, Sofia, Bulgaria. ${ }^{5}$ Twin Cities Orthopedics, Edina, Minnesota, USA. ${ }^{6}$ Department of Normal and Clinical Anatomy, Interfaculty Chair of Anatomy and Histology, Medical University of Lodz, Lodz, Poland. 7 Department of Anatomical Sciences, St. George's University, True Blue, Grenada. ${ }^{8}$ Department of Neurosurgery, Tulane University School of Medicine, New Orleans, Louisiana, USA. ${ }^{9}$ Department of Neurology, Tulane University School of Medicine, New Orleans, Louisiana, USA. ${ }^{10}$ Department of Structural and Cellular Biology, Tulane University School of Medicine, New Orleans, Louisiana, USA. ${ }^{11}$ Department of Surgery, Tulane University School of Medicine, New Orleans, USA.

Received: 25 August 2021 Accepted: 6 December 2021

Published online: 14 January 2022

\section{References}

1. Andrews K, Lu A, Mckean L, Ebraheim N (2017) Review: medial collateral ligament injuries. J Orthop 14(4):550-554

2. DeGrace DM, Gill TJ IV, Gill TJ III (2013) Analysis of medial collateral ligament injuries of the knee. Harvard Orthop J 15:13-24

3. Woo SL, Abramowitch SD, Kilger R, Liang R (2006) Biomechanics of knee ligaments: injury, healing, and repair. J Biomech 39(1):1-20

4. Zhang J, Pan T, Im HJ, Fu FH, Wang JH (2011) Differential properties of human $\mathrm{ACL}$ and $\mathrm{MCL}$ stem cells may be responsible for their differential healing capacity. BMC Med 9:68

5. Wijdicks CA, Griffith CJ, Johansen S, Engebretsen L, LaPrade RF (2010) Injuries to the medial collateral ligament and associated medial structures of the knee. J Bone Joint Surg Am 92(5):1266-1280

6. Georgiev GP, Landzhov B, Dimitrova IN, Slavchev S, Malinova L, Kartelov $Y$ et al (2015) Light microscopic and immunohistochemical study of the medial collateral ligament epiligament in rat knee. Compt Rend Acad Bulg Sci 68(1):95-100

7. Georgiev GP, Landzhov B, Dimitrova IN, Slavchev S, Malinova L, Ovtscharoff W (2015) Immunohistochemical study during early healing of the medial collateral ligament epiligament in rat knee model. Compt Rend Acad Bulg Sci 68(5):655-660

8. Georgiev GP, Iliev A, Kotov G, Kinov P, Slavchev S, Landzhov B (2017) Light and electron microscopic study of the medial collateral ligament epiligament tissue in human knees. World J Orthop 8(5):372-378

9. Chamberlain CS, Crowley EM, Kobayashi H, Eliceiri KW, Vanderby R (2011) Quantification of collagen organization and extracellular matrix factors within the healing ligament. Microsc Microanal 17(5):779-787

10. Creighton RA, Spang JT, Dahners LE (2005) Basic science of ligament healing: medial collateral ligament healing with and without treatment. Sports Med Arthrosc Rev 13(3):145-150 
11. Georgiev GP, Vidinov NK, Kinov PS (2010) Histological and ultrastructural evaluation of the early healing of the lateral collateral ligament epiligament tissue in a rat knee model. BMC Musculoskelet Disord 11:117

12. Georgiev GP, Kotov G, lliev A, Kinov P, Angelova J, Landzhov B (2018) Comparison between operative and non-operative treatment of the medial collateral ligament: histological and ultrastructural findings during early healing in the epiligament tissue in a rat knee model. Cells Tissues Organs 206(3):165-182

13. Jia ZY, Zhang C, Cao SQ, Xue CC, Liu TZ, Huang X et al (2017) Comparison of artificial graft versus autograft in anterior cruciate ligament reconstruction: a meta-analysis. BMC Musculoskelet Disord 18(1):309

14. Kiapour AM, Murray MM (2014) Basic science of anterior cruciate ligament injury and repair. Bone Joint Res 3(2):20-31

15. Kim HS, Seon JK, Jo AR (2013) Current trends in anterior cruciate ligament reconstruction. Knee Surg Relat Res 25(4):165-173

16. Vaishya R, Agarwal AK, Ingole S, Vijay V (2015) Current trends in anterior cruciate ligament reconstruction: a review. Cureus 7(11):e378

17. Majima T, Lo IK, Marchuk LL, Shrive NG, Frank CB (2006) Effects of ligament repair on laxity and creep behavior of an early healing ligament scar. J Orthop Sci 11(3):272-277

18. Georgiev GP, Landzhov B, Dimitrova IN, Malinova L, OvtscharoffW (2016) Expression of fibronectin during early healing of the medial collateral ligament epiligament in rat knee model. Compt Rend Acad Bulg Sci 69(5):639-644

19. Georgiev GP, Iliev A, Landzhov B, Dimitrova IN, Kotov G, Malinova L et al (2017) Localization of matrix metalloproteinase-2 in injured medial collateral ligament epiligament in rat knee. Compt Rend Acad Bulg Sci 70(2):273-278

20. Georgiev GP, Landzhov B, Kotov G, Slavchev SA, Iliev A (2018) Matrix metalloproteinase-2 and -9 expression in the epiligament of the medial collateral and anterior cruciate ligament in human knees: a comparative study. Cureus 10(11):e3550

21. Georgiev GP, Iliev A, Kotov G, Nedialkova VK, Kirkov V, Landzhov B (2019) Epiligament tissue of the medial collateral ligament in rat knee joint: ultrastructural study. Cureus 11(1):e3812

22. Georgiev GP, Kotov G, Iliev A, Slavchev S, Ovtscharoff W, Landzhov B (2019) A comparative study of the epiligament of the medial collateral and the anterior cruciate ligament in the human knee. Immunohistochemical analysis of collagen type I and V and procollagen type III. Ann Anat 224:88-96

23. Kamawal Y, Steinert AF, Holzapfel BM, Rudert M, Barthel T (2016) Case report - calcification of the medial collateral ligament of the knee with simultaneous calcifying tendinitis of the rotator cuff. BMC Musculoskelet Disord 17:283

24. LaPrade RF, Engebretsen AH, Ly TV, Johansen S, Wentorf FA, Engebretsen $L$ (2007) The anatomy of the medial part of the knee. J Bone Joint Surg Am 89(9):2000-2010

25. Nguyen DT, Ramwadhdoebe TH, van der Hart CP, Blankevoort L, Tak PP, van Dijk CN (2014) Intrinsic healing response of the human anterior cruciate ligament: an histological study of reattached $A C L$ remnants. J Orthop Res 32(2):296-301

26. Amis AA (2012) The functions of the fibre bundles of the anterior cruciate ligament in anterior drawer, rotational laxity and the pivot shift. Knee Surg Sports Traumatol Arthrosc 20(4):613-620

27. Sonnery-Cottet B, Bazille C, Hulet C, Colombet P, Cucurulo T, Panisset JCet al. (2014) French arthroscopic society. Histological features of the ACL remnant in partial tears. Knee 21(6):1009-1013

28. Ziegler CG, Pietrini SD, Westerhaus BD, Anderson CJ, Wijdicks CA, Johansen S et al (2011) Arthroscopically pertinent landmarks for tunnel positioning in single-bundle and double-bundle anterior cruciate ligament reconstructions. Am J Sports Med 39(4):743-752

29. Frank CB (2004) Ligament structure, physiology and function. J Musculoskelet Neuronal Interact 4(2):199-201

30. Hsu SL, Liang R, Woo SL (2010) Functional tissue engineering of ligament healing. Sports Med Arthrosc Rehabil Ther Technol 2:12

31. Wan C, Hao Z, Wen S (2013) A quantitative comparison of morphological and histological characteristics of collagen in the rabbit medial collateral ligament. Ann Anat 195(6):562-569

32. Iliev A, Kotov G, Stamenov N, Landzhov B, Kirkov V, Georgiev GP (2021) A comparative immunohistochemical and quantitative study of the epiligament of the medial collateral and anterior cruciate ligament in rat knee. Int J Morphol 39(1):151-159

33. Chowdhury P, Matyas JR, Frank CB (1991) The "epiligament" of the rabbit medial collateral ligament: a quantitative morphological study. Connect Tissue Res 27(1):33-50

34. Hauser RA, Dolan EE, Phillips HJ, Newlin AC, Moore RE, Woldin BA (2013) Ligament injury and healing: a review of current clinical diagnostics and therapeutics. Open Rehab J 6:1-20

35. Niyibizi C, Kavalkovich K, Yamaji T, Woo SL (2000) Type V collagen is increased during rabbit medial collateral ligament healing. Knee Surg Sports Traumatol Arthrosc 8(5):281-285

36. Yang L, Tsai CM, Hsieh AH, Lin VS, Akeson WH, Sung KL (1999) Adhesion strength differential of human ligament fibroblasts to collagen types I and III. J Orthop Res 17(5):755-762

37. Amiel D, Kleiner JB, Roux RD, Harwood FL, Akeson WH (1986) The phenomenon of "ligamentization": anterior cruciate ligament reconstruction with autogenous patellar tendon. J Orthop Res 4(2):162-172

38. Breuls RG, Klumpers DD, Everts V, Smit TH (2009) Collagen type V modulates fibroblast behavior dependent on substrate stiffness. Biochem Biophys Res Commun 380(2):425-429

39. Junqueira LC, Carneiro J, Kelley RO (1998) Basic histology. In: Connective tissue. Lange Medical Books/McGraw-Hill, New York

40. Bray RC, Leonard CA, Salo PT (2003) Correlation of healing capacity with vascular response in the anterior cruciate and medial collateral ligaments of the rabbit. J Orthop Res 21(6):1118-1123

41. Georgiev GP (2021) Epiligament or paratenon is more appropriate for describing the enveloping tissue of the cruciate ligaments of the human knee? Folia Morphol (Warsz). https://doi.org/10.5603/FM.a2021.0021

42. O'Donoghue DH, Rockwood CA, Zaricznyj B, Kenyon R (1961) Repair of knee ligaments in dogs. I The lateral collateral ligament. J Bone Joint Surg Am 43:1167-1178

43. Hart DP, Dahners LE (1987) Healing of the medial collateral ligament in rats. The effects of repair, motion, and secondary stabilizing ligaments. J Bone Joint Surg Am 69(8):1194-1199

44. Hildebrand KA, Frank CB (1998) Scar formation and ligament healing. Can J Surg 41(6):425-429

45. Chimich D, Frank C, Shrive N, Dougall H, Bray R (1991) The effects of initial end contact on medial collateral ligament healing: a morphological and biomechanical study in a rabbit model. J Orthop Res 9(1):37-47

46. Loitz-Ramage BJ, Frank CB, Shrive NG (1997) Injury size affects long-term strength of the rabbit medial collateral ligament. Clin Orthop Relat Res 337:272-280

47. McDougall JJ, Yeung G, Leonard CA, Sutherland C, Bray RC Adaptation of post-traumatic angiogenesis in the rabbit knee by apposition of torn ligament ends. J Orthop Res 18(4):663-670

48. Ishiguro N, Shimizu T, Ito T, Kojima T, Iwahori Y, Iwata H (2000) The expression of matrix metalloproteinases and inhibitors in acute rupture of the anterior cruciate ligament. Mod Rheumatol 10(2):95-102

49. Zhou D, Lee HS, Villarreal F, Teng A, Lu E, Reynolds S et al (2005) Differential MMP-2 activity of ligament cells under mechanical stretch injury: an in vitro study on human ACL and MCL fibroblasts. J Orthop Res 23(4):949-957

50. Tang Z, Yang L, Xue R, Zhang J, Wang Y, Chen PC et al (2009) Differential expression of matrix metalloproteinases and tissue inhibitors of metalloproteinases in anterior cruciate ligament and medial collateral ligament fibroblasts after a mechanical injury: involvement of the p65 subunit of NF-kappaB. Wound Repair Regen 17(5):709-716

51. Zhang J, Yang L, Tang Z, Xue R, Wang Y, Luo Z et al (2009) Expression of MMPs and TIMPs family in human ACL and MCL fibroblasts. Connect Tissue Res 50(1):7-13

52. Andrish J, Holmes R (1979) Effects of synovial fluid on fibroblasts in tissue culture. Clin Orthop Relat Res 138:279-283

53. Lyon RM, Akeson WH, Amiel D, Kitabayashi LR, Woo SL (1991) Ultrastructural differences between the cells of the medical collateral and the anterior cruciate ligaments. Clin Orthop Relat Res 272:279-286

54. Yoshida M, Fujii K (1999) Differences in cellular properties and responses to growth factors between human ACL and MCL cells. J Orthop Sci 4(4):293-298

55. Cao M, Stefanovic-Racic M, Georgescu HI, Fu FH, Evans CH (2000) Does nitric oxide help explain the differential healing capacity of the anterior 
cruciate, posterior cruciate, and medial collateral ligaments? Am J Sports Med 28(2):176-182

56. Bray RC, Fisher AW, Frank CB (1990) Fine vascular anatomy of adult rabbit knee ligaments. J Anat 172:69-79

57. Nishikawa Y, Kokubun T, Kanemura N, Takahashi T, Matsumoto M, Maruyama $\mathrm{H}$ et al (2018) Effects of controlled abnormal joint movement on the molecular biological response in intra-articular tissues during the acute phase of anterior cruciate ligament injury in a rat model. BMC Musculoskelet Disord 19(1):175

58. Chen CH (2009) Graft healing in anterior cruciate ligament reconstruction. Sports Med Arthrosc Rehabil Ther Technol 1(1):21

59. Vavken P, Murray MM (2011) The potential for primary repair of the ACL. Sports Med Arthrosc Rev 19(1):44-49

\section{Publisher's Note}

Springer Nature remains neutral with regard to jurisdictional claims in published maps and institutional affiliations.

\section{Submit your manuscript to a SpringerOpen ${ }^{\circ}$ journal and benefit from:}

- Convenient online submission

- Rigorous peer review

- Open access: articles freely available online

- High visibility within the field

- Retaining the copyright to your article

Submit your next manuscript at $\boldsymbol{\nabla}$ springeropen.com 